\title{
Radical radiotherapy for localized cutaneous angiosarcoma of the scalp
}

\author{
ATSUTO KATANO, HIDEOMI YAMASHITA and KEIICHI NAKAGAWA \\ Department of Radiology, The University of Tokyo Hospital, Bunkyo-ku, Tokyo 113-8655, Japan
}

Received December 23, 2020; Accepted June 16, 2021

DOI: $10.3892 / \mathrm{mco} .2021 .2357$

\begin{abstract}
Angiosarcoma is a rare but highly aggressive sarcoma of mesenchymal origin with a high mortality rate. Due to its rarity, there are very limited reports on the clinical outcomes of angiosarcoma treated with radical radiotherapy. The aim of the present study was to evaluate the efficacy and feasibility of treating patients with radiotherapy for cutaneous angiosarcoma localized to the scalp at The University of Tokyo Hospital (Tokyo, Japan). The present study analyzed 15 consecutive patients treated for cutaneous angiosarcoma of the scalp with radiotherapy between June 2008 and January 2020. All patients were treated with 70 Gy of irradiation split into 35 fractions, focused on the lesion, including 9 patients who received total scalp radiotherapy. The median follow-up period in all patients was 9.7 months. The median overall survival (OS) time was 20.7 months, and the 1-, 2- and 5-year OS rates were $56.2,28.1$ and $9.4 \%$, respectively. At the time of analysis, 13 patients $(86.7 \%)$ developed recurrence. Among these 13 patients, the first site of recurrence was the scalp as local recurrence in 7 patients $(46.7 \%)$, parotid recurrence in 2 patients $(13 \%)$ and distant metastasis in 4 patients $(26.7 \%)$. No patient exhibited grade 3-5 radiation-induced late toxicity. Therefore, the present study revealed the clinical outcomes of radical radiotherapy for cutaneous angiosarcoma of the scalp.
\end{abstract}

\section{Introduction}

Cutaneous angiosarcoma (CAS) is a rare neoplasm of mesenchymal origin, accounting for less than $1 \%$ of all sarcomas (1). While the pathogenesis of angiosarcoma remains unclear, it has been associated with multiple etiological risk factors, including radiotherapy, chronic lymphedema, various chemicals, and immunodeficiency (2). In a population-based

Correspondence to: Dr Hideomi Yamashita, Department of Radiology, The University of Tokyo Hospital, 7-3-1 Hongo, Bunkyo-ku, Tokyo 113-8655, Japan

E-mail: yamachan07291973@yahoo.co.jp

Key words: angiosarcoma, scalp, radiotherapy, treatment outcome, retrospective analysis retrospective cohort study, radiotherapy significantly increased the risk of angiosarcoma (3). Angiosarcoma arising in the base of chronic lymphedema is known as Stewart-Treves syndrome. Some investigators have reported a relationship between antecedent traumatic tissue injury and pathogenesis (4). Recently, ultraviolet light exposure has been associated with a common etiological and genomic mutational basis for the pathogenesis of angiosarcoma of the head and neck region (5).

The most commonly affected sites of CAS are the scalp and facial skin (6), which are associated with a poor prognosis compared to other sites (7). The clinical presentation of CAS is single or multiple bluish or violaceous nodules, and occasionally ulceration or bleed (8). No definitive criteria have yet been formulated for the staging of angiosarcoma. According to the 8th edition of the American Joint Committee on Cancer staging manual on the TNM staging system, angiosarcoma is excluded from the soft tissue tumor chapter because of its aggressive infiltrative nature. On immunohistochemistry, vascular endothelial markers, including CD31, CD34, and von Willebrand factor-related antigens, are informative for diagnosis (9). In addition, lymphatic endothelial markers, such as D2-40, Prox-1, and VEGFR-3, might be positively expressed, which indicates lymphatic differentiation of angiosarcoma (10).

Although several treatment procedures have been investigated, as yet, there is no consensus on the treatment of angiosarcoma, due to a lack of statistically significant evidence. Surgical resection with a free tumor margin is the primary treatment of choice for angiosarcoma (11). The microscopic negative surgical margin was significantly correlated with longer overall survival (OS), which is considered an important prognostic factor (12). However, due to the diffuse infiltrative and multifocal nature of CAS, preoperative medical assessment of the lesion and careful selection of the patient are critically important.

Radiotherapy is also considered a curative local therapy for unresectable or incompletely resected tumors. The effectiveness of postoperative radiotherapy for local control (LC) and OS has been reported in several studies (13) however studies on the treatment of angiosarcoma by radical radiotherapy without surgery are very limited. The aim of the present study was to investigate the clinical outcomes of radiotherapy without surgery for patients with scalp CAS. 


\section{Materials and methods}

Inclusion and exclusion criteria of the patients. We conducted a retrospective analysis of consecutive patients with scalp-localized CAS treated with radical radiotherapy at the Department of Radiology in our institution from June 2008 to January 2020. This retrospective study was performed in accordance with the guidelines approved by the institutional review board (ID number: 3372). All patients provided written informed consent. The patients in this study satisfied the following inclusion criteria: a) histologically confirmed angiosarcoma located in the scalp, b) treated with radiotherapy with curative intent, c) no distant metastasis, and d) no history of previous radiotherapy or surgery of the scalp lesion. Retrospective patient data were obtained from the medical records of our institution. Performance status of the patients was assessed by Karnofsky Performance Status (14).

Radiation treatment planning. All patients underwent either electron beam radiotherapy or helical tomotherapy-based intensity-modulated radiotherapy (HT-IMRT). The radiation field design was either the partial scalp or whole scalp, which was decided by a clinician at the time of treatment. For partial scalp treatment, a 6-10 MeV electron radiotherapy beam with a 5-mm bolus was administered to the primary site with generous margins. In the HT-IMRT treatment group, the planning computed tomography (CT) dataset was acquired with a thermoplastic mask on a flat board for planning CT. The CT image data were reconstructed with a slice thickness of $2 \mathrm{~mm}$. These data were then sent to a treatment planning system, such as Pinnacle (Philips), Monaco (Elekta CMS), or TomoTherapy Planning Station (Accuray). The clinical target volume (CTV) included the primary tumor and total scalp. The planning target volume (PTV) included the CTV with a 3-mm margin only on the inside of the CTV and excluding the area outside the CTV. The prescribed dose was 70 Gy spread across 35 fractions targeted to $95 \%$ (exceeding $95 \%$ of the volume) of the PTV. The treatment planning used a virtual bolus to avoid hot spot doses; this process has been described in detail in a previous report from our institution (15).

Statistical analysis. Data analysis was performed using the $\mathrm{R}$ Statistical Software (Foundation for Statistical Computing). OS, progression-free survival (PFS), LC, and distant metastasis control (DMC) rates were calculated from the first day of radiotherapy using the Kaplan-Meier method. OS was defined as the time interval until death from any cause, and surviving patients were censored at the date of the last follow-up examination. PFS was defined as the time interval until progression or death from any cause, and living patients without disease progression were censored at the date of the last follow-up examination. LC was defined as the time interval until intra-scalp recurrence, and patients free from local recurrence were censored at the date of the last follow-up examination or death. DMC was defined as the time interval until metastatic recurrence, and patients free from metastatic recurrence were censored at the date of the last follow-up examination or death. Univariate Cox hazard analysis was used to calculate the hazard ratios of the factors associated with OS and PFS. Statistical significance was set at $\mathrm{P}<0.05$.
Table I. Characteristics of 15 patients with cutaneous angiosarcoma of the scalp treated by definitive radiotherapy.

\begin{tabular}{lc}
\hline Variables & Value \\
\hline Age, years (median; range) & $75(59-84)$ \\
Sex, n (\%) & $14(93)$ \\
Male & $1(7)$ \\
Female & \\
Karnofsky Performance Status, n (\%) & $1(7)$ \\
100 & $10(67)$ \\
90 & $4(27)$ \\
80 & \\
Radiotherapy, n (\%) & $6(40)$ \\
Partial scalp (electron) & $2(13)$ \\
Total scalp (electron) & $7(47)$ \\
Total scalp (tomotherapy) & \\
Concurrent systemic therapy, n (\%) & $1(7)$ \\
rIL-2 & $2(13)$ \\
DOC & $6(40)$ \\
PTX & $5(33)$ \\
DOC + rIL-2 & $1(7)$ \\
PTX + rIL-2 & \\
Adjuvant therapy, n (\%) & $1(7)$ \\
rIL-2 injection & $8(53)$ \\
DOC & $3(20)$ \\
PTX & $3(20)$ \\
None &
\end{tabular}

rIL-2, recombinant interleukin-2; DOC, docetaxel; PTX, paclitaxel.

\section{Results}

Background of patients. Fifteen patients were retrospectively analyzed in this study; patient characteristics are shown in Table I. The median age was 75 years (range, 59-84 years), and the majority (93.3\%) of patients were male. Six patients received partial radiotherapy, while the others received total scalp radiotherapy. All but one patient received radiotherapy with concurrent taxane regimen chemotherapy: Weekly paclitaxel (7 patients, $46.7 \%$ ) or 3-weekly docetaxel (7 patients, 46.7\%). Intravenous administration of recombinant interleukin-2 (rIL-2) was administered to six patients (40.0\%). Adjuvant therapy was administered to 12 patients. The monthly docetaxel regimen was administered to eight patients, weekly paclitaxel regimen to three patients, and local intralesional injection to one patient.

Survival analysis. The median follow-up period for these patients was 9.7 months (range: 3.5-72.8 months). At the time of analysis, one patient was lost to follow-up; one was alive with active disease; one was alive with controlled disease; and the other 12 patients had died, one of whom died from a myocardial infarction, which was not directly associated with primary disease or its treatment. The median OS time was 20.7 months [95\% confidence interval (CI): 7.6-48.3 months], and the 1-, 3- and 5-year OS rates were 56.2, 28.1 and 9.4\%, 
Table II. Risk factors for overall survival and progression-free survival time according to univariate Cox hazard analysis.

\begin{tabular}{lllll}
\hline & \multicolumn{2}{c}{ Overall survival } & & Progression-free survival \\
\cline { 2 - 3 } Covariables & Hazard ratio $(95 \% \mathrm{CI})$ & P-value & Hazard ratio (95\% CI) & P-value \\
\hline Age (<75 years vs. $\geq 75$ years) & $1.672(0.449-6.221)$ & 0.444 & $1.948(0.579-6.556)$ \\
Performance status (>80\% vs. $\leq 80 \%)$ & $3.788(0.928-15.470)$ & 0.064 & $2.870(0.751-10.960)$ & 0.281 \\
Radiation scalp field (partial vs. total) & $4.993(0.929-26.850)$ & 0.061 & $3.678(0.927-14.590)$ & 0.064 \\
Chemotherapy regimen (weekly PTX vs. others) & $0.292(0.053-1.620)$ & 0.159 & $0.355(0.105-1.204)$ & 0.097 \\
rIL-2 administration (yes vs. no) & $3.449(0.781-15.230)$ & 0.102 & $2.672(0.756-9.445)$ \\
Adjuvant therapy (yes vs. no) & $3.391(0.614-18.730)$ & 0.161 & $3.259(0.534-19.890)$ & 0.127 \\
\hline
\end{tabular}

95\% CI, 95\% confidence interval; PTX, paclitaxel; rIL-2, recombinant interleukin-2.
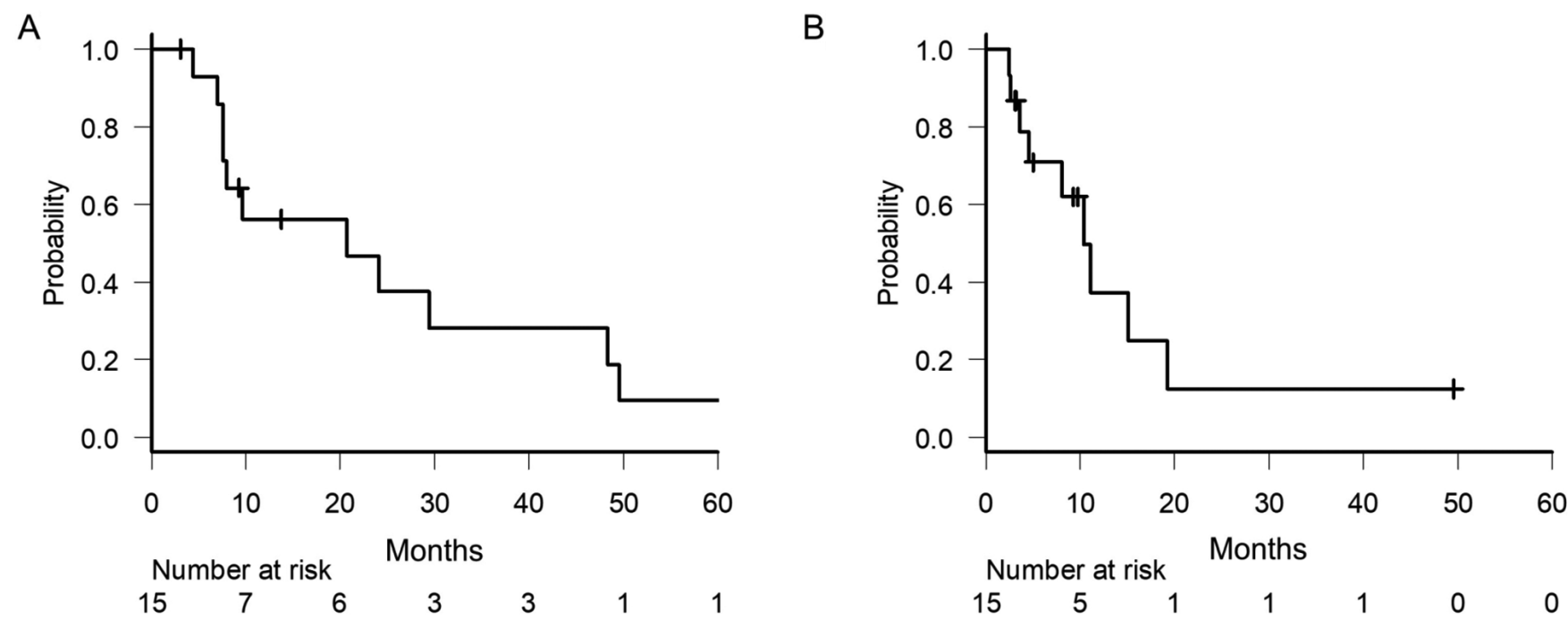

Figure 1. Data of the 15 patients with cutaneous angiosarcoma treated by radiotherapy. (A) Kaplan-Meier plot of overall survival rate. (B) Kaplan-Meier plot of progression-free survival rate. A small vertical line denotes a censored patient.

respectively (Fig. 1A). The median PFS time was 9.3 months (95\% CI: 3.3-11.0 months), and the 1- and 3-year PFS rates were 21.7 and $7.2 \%$, respectively (Fig. 1B). The factors associated with favorable OS or PFS were not identified in the univariate analysis (Table II).

Recurrence and salvage treatment. At the time of analysis, 13 patients $(86.7 \%)$ developed recurrence. Among these, the first site of recurrence was the scalp, as local recurrence in seven patients $(46.7 \%)$, parotid recurrence in two (13\%), and distant metastasis in four (26.7\%). The 1-year LC and DMC rates were 37.8 and $61.5 \%$, respectively. In the seven patients with local recurrence, salvage surgery was performed in one patient, additional electron radiotherapy in four patients, and best supportive care in two patients. Two patients with parotid recurrence were treated with EBRT. Among the patients with distant metastasis, one received salvage chemotherapy (weekly PTX) and the others selected the best supportive care.

Adverse event. The most common radiation-induced acute adverse event was skin reaction. Moderate (G2) or severe (G3) acute skin reactions were present in all patients, consisting of 12 patients in G2 and three in G3. Severe (G3 or higher) radiation-induced late complications, such as fistulas, strictures, or necrosis of the bone, were not observed.

\section{Discussion}

The results of our study did not deviate significantly from those of other studies. The clinical outcomes of patients in our study were comparable to those of previous reports. Hata et al (16) reported that 17 patients with angiosarcoma of the scalp underwent total scalp irradiation therapy with curative intent and found that the 3-year OS and disease-free survival rates for all patients were 22 and $6 \%$, respectively. They adopted a two-step cone-down technique, with an initial phase CTV including the entire scalp for a median dose of 50 Gy in 25 fractions, followed by a tumor site with an additional margin for a dose of $20 \mathrm{~Gy}$ in 10 fractions. However, five patients experienced disease progression in the scalp distant from the primary site, which had received prophylactic irradiation at doses of 46-50 Gy. They, therefore, concluded that a dose of less than 50 Gy in conventional fractions might 
be insufficient to control microscopic tumors. Clear evidence to determine the effective radiation dose and coverage field is debated, and the optimal treatment strategy for radiotherapy remains controversial.

Multimodal treatments, including surgery and radiotherapy, were more effective than single-modality treatment in improving clinical outcomes for patients with CAS. Ogawa et al (17) revealed that patients treated with combined therapy had a significantly more favorable OS than patients treated with either surgery or radiotherapy alone (2-year OS: $45.8 \%$ vs. $11.1 \%, \mathrm{P}<0.001)$. Guadagnolo et al (18) also reported that combined modality local therapy was associated with improvement in LC, OS, and disease-specific survival in 70 patients with angiosarcoma of the face or scalp.

The role of adjuvant systemic therapy after initial treatment has been investigated in previous retrospective studies. Ihara et al (19) reported that the administration of adjuvant chemotherapy consisting of a taxane regimen after concurrent chemoradiotherapy was a significant prognostic factor for PFS $(\mathrm{P}=0.036)$. Fujisawa et al $(20)$ showed that patients who received taxane-based concurrent chemoradiotherapy (CCRT) with maintenance chemotherapy showed a significant improvement in OS compared to those receiving CCRT alone $(\mathrm{P}<0.01)$. In the present study, while the OS and PFS were superior in the maintenance chemotherapy group, the difference was not statistically significant.

The most frequent metastatic site of angiosarcoma is the lungs (21), as shown in our report. Treatment options for the management of recurrent or advanced CAS are limited. However, in recent years, immunotherapeutic approaches have emerged as promising anti-cancer systemic therapies. In particular, immune checkpoint inhibitors have proven efficacious in various cancer entities. Florou et al (22) treated seven angiosarcoma patients with immune checkpoint inhibitors, such as anti-cytotoxic T-lymphocyte antigen 4 or programmed cell death protein 1 monoclonal antibody, five (71\%) of whom had a partial response, without severe toxicity.

In the present study, no statistically significant prognostic factors were identified. However, local recurrence and distant metastasis, especially in the lungs, were observed at a considerable frequency. These pulmonary metastases could cause fatal hemopneumothorax, and careful follow-up for distant metastasis, including lung metastasis, after treatment is considered crucial.

This study had several limitations. As the study was conducted at a single institution, the sample size was small, making it difficult to identify significant prognostic factors from the data. Because of the retrospective nature of data collection, our data had numerous risks of bias. Moreover, the retrospective analysis of medical records makes it impossible to describe the complete condition of patients at the time of treatment.

This retrospective study reported the clinical outcomes of radiotherapy for CAS in our institution. Radiotherapy combined with adjuvant chemotherapy showed a more favorable outcome than radiotherapy alone; however, there were no statistically significant differences between these groups. Further comprehensive research is needed to clarify the optimal treatment strategy for CAS.

\section{Acknowledgements}

Not applicable.

\section{Funding}

No funding was received.

\section{Availability of data and materials}

All data generated or analyzed during this study are included in this published article.

\section{Authors' contributions}

AK performed acquisition of data. $\mathrm{HY}$ and $\mathrm{KN}$ analyzed and interpreted the clinical data. $\mathrm{KN}, \mathrm{AK}$ and $\mathrm{HY}$ conceived the study and participated in its design and coordination. AK was a major contributor to the writing of this report. $\mathrm{KN}$ and $\mathrm{HY}$ critically revised this report for important intellectual content. The authenticity of all the raw data was confirmed by HY and AK. All authors read and approved the final manuscript.

\section{Ethics approval and consent to participate}

The present study was approved by the Institutional Review Board of The University of Tokyo Hospital. Written informed consent was obtained from all patients.

\section{Patient consent for publication}

Not applicable.

\section{Competing interests}

The authors declare that they have no competing interests.

\section{References}

1. Conic RRZ, Damiani G, Frigerio A, Tsai S, Bragazzi NL, Chu TW, Mesinkovska NA, Koyfman SA, Joshi NP, Budd GT, et al: Incidence and outcomes of cutaneous angiosarcoma: A SEER population-based study. J Am Acad Dermatol 83: 809-816, 2020.

2. Young RJ, Brown NJ, Reed MW, Hughes D and Woll PJ: Angiosarcoma. Lancet Oncol 11: 983-991, 2010.

3. Huang J and Mackillop WJ: Increased risk of soft tissue sarcoma after radiotherapy in women with breast carcinoma. Cancer 92: 172-180, 2001.

4. Meis-Kindblom JM and Kindblom LG: Angiosarcoma of soft tissue: A study of 80 cases. Am J Surg Pathol 22: 683-697, 1998.

5. Painter CA, Jain E, Tomson BN, Dunphy M, Stoddard RE, Thomas BS, Damon AL, Shah S, Kim D, Gómez Tejeda Zañudo J, et al: The angiosarcoma project: Enabling genomic and clinical discoveries in a rare cancer through patient-partnered research. Nat Med 26: 181-187, 2020

6. Gaballah AH, Jensen CT, Palmquist S, Pickhardt PJ, Duran A, Broering G and Elsayes KM: Angiosarcoma: Clinical and imaging features from head to toe. Br J Radiol 90: 20170039, 2017.

7. Wang L, Lao IW, Yu L and Wang J: Clinicopathological features and prognostic factors in angiosarcoma: A retrospective analysis of 200 patients from a single Chinese medical institute. Oncol Lett 14: 5370-5378, 2017.

8. Shon W and Billings SD: Cutaneous malignant vascular neoplasms. Clin Lab Med 37: 633-646, 2017.

9. Ohsawa M, Naka N, Tomita Y, Kawamori D, Kanno H and Aozasa K: Use of immunohistochemical procedures in diagnosing angiosarcoma. Evaluation of 98 cases. Cancer 75: 2867-2874, 1995. 
10. Mankey CC, McHugh JB, Thomas DG and Lucas DR: Can lymphangiosarcoma be resurrected? A clinicopathological and immunohistochemical study of lymphatic differentiation in 49 angiosarcomas. Histopathology 56: 364-371, 2010.

11. Morgan MB, Swann M, Somach S, Eng W and Smoller B: Cutaneous angiosarcoma: A case series with prognostic correlation. J Am Acad Dermatol 50: 867-874, 2004.

12. Fury MG, Antonescu CR, Van Zee KJ, Brennan MF and Maki RG: A 14-year retrospective review of angiosarcoma: Clinical characteristics, prognostic factors, and treatment outcomes with surgery and chemotherapy. Cancer J 11: 241-247, 2005.

13. Pawlik TM, Paulino AF, McGinn CJ, Baker LH, Cohen DS, Morris JS, Rees R and Sondak VK: Cutaneous angiosarcoma of the scalp: A multidisciplinary approach. Cancer 98: 1716-1726, 2003.

14. Karnofsky DA and Burchenal JH: Evaluation of chemotherapeutic agents. In: The Clinical Evaluation of Chemotherapeutic Agents in Cancer. MacLeod CM, editor. Columbia University Press, New York, pp191-205, 1949.

15. Takenaka R, Haga A, Nawa K, Hideomi Y and Nakagawa K Improvement of the robustness to set up error by a virtual bolus in total scalp irradiation with Helical tomotherapy. Radiol Phys Technol 12: 433-437, 2019.

16. Hata M, Wada H, Ogino I, Omura M, Koike I, Tayama Y Odagiri K, Kasuya $\mathrm{T}$ and Inoue T: Radiation therapy for angiosarcoma of the scalp: Treatment outcomes of total scalp irradiation with X-rays and electrons. Strahlenther Onkol 190: 899-904, 2014.
17. Ogawa K, Takahashi K, Asato Y, Yamamoto Y, Taira K, Matori S, Iraha S, Yagi N, Yogi A, Haranaga S, et al: Treatment and prognosis of angiosarcoma of the scalp and face: A retrospective analysis of 48 patients. Br J Radiol 85: e1127-e1133, 2012.

18. Guadagnolo BA, Zagars GK, Araujo D, Ravi V, Shellenberger TD and Sturgis EM: Outcomes after definitive treatment for cutaneous angiosarcoma of the face and scalp. Head Neck 33: 661-667, 2011.

19. Ihara H, Kaji T, Katsui K, Miyake T, Waki T, Katayama N, Matsuzaki H, Yamasaki O, Kuroda M, Morizane S and Kanazawa S: Single institutional experience of radiation therapy for angiosarcoma of the scalp without cervical lymph node metastases: Impact of concurrent chemoradiation with maintenance chemotherapy using taxanes on patient prognosis. Mol Clin Oncol 11: 498-504, 2019.

20. Fujisawa Y, Yoshino K, Kadono T, Miyagawa T, Nakamura Y and Fujimoto M: Chemoradiotherapy with taxane is superior to conventional surgery and radiotherapy in the management of cutaneous angiosarcoma: A multicentre, retrospective study. Br J Dermatol 171: 1493-1500, 2014.

21. Mark RJ, Poen JC, Tran LM, Fu YS and Juillard GF: Angiosarcoma. A report of 67 patients and a review of the literature. Cancer 77: 2400-2406, 1996.

22. Florou V, Rosenberg AE, Wieder E, Komanduri KV, Kolonias D, Uduman M, Castle JC, Buell JS, Trent JC and Wilky BA: Angiosarcoma patients treated with immune checkpoint inhibitors: A case series of seven patients from a single institution. J Immunother Cancer 7: 213, 2019. 